\title{
Socioeconomic predictors of adherence behavior among HIV-positive patients receiving antiretroviral therapy in Selangor, Malaysia
}

\begin{abstract}
Medication adherence remains a critical link between the prescribed ART regimen and treatment outcome. Several factors may influence adherence behavior. This cross-sectional study aimed to highlight socioeconomic predictors of adherence behavior among a cohort of 242 adult Malaysian patients receiving antiretroviral therapy in Hospital Sungai Buloh, Malaysia, where they were enrolled in a parent study (single-blinded randomized controlled trial) between January and December 2014. Statistical analysis of secondary data on adherence behavior and sociodemographic characteristics of the patients revealed mean age of 33.4 years and ranged from 18 to 64 years; $88.8 \%$ were males. A total of 224 (93\%) patients who completed 6 months' adherence assessment were included in the model. Of these, $135(60.3 \%)$ achieved optimal adherence. Multivariate binary logistic regression analysis revealed that patient's income and ethnicity were significant predictors of adherence behavior. This may be valuable for targeted programmatic interventions to further enhance successful treatment outcomes among the target population.
\end{abstract}

Keyword: Adherence; Antiretroviral therapy (ART); Behavior; Predictors; Socioeconomic 
